\title{
ESTUDIO DE LAS CATEGORÍAS VACÍAS, pro Y PRO: APLICACIÓN DE LA RCG EN ESPANOL
}

HUI-CHUAN LU

National Cheng Kung University

\section{RESUMEN}

El presente trabajo señala que el español, que es una lengua en la que existen ambas categorías vacías, pro y PRO, puede, por un lado, ofrecer pruebas que apoyen la viabilidad de la Regla de Control Generalizado Revisado propuesta por Huang (1989). Sin embargo, por otro lado, la lengua española causa problemas en los análisis de Rosenbaum (1967) y de Huang (1989), donde se intentan distinguir los verbos decir y tratar, atendiendo a sus características verbales. Por consiguiente, o bien dichos análisis necesitan ser modificados, o bien es necesario propugnar que la forma verbal decir, en español, presenta un comportamiento divergente al que manifiesta en otras lenguas. Además, modificamos el Principio de Identificación de Jaeggeli (1989) y aplicamos la Regla de Interpretación Arbitraria propuesta por Rizzi (1986) para resolver los problemas relacionados con el control libre y la interpretación arbitraria de las categorías vacias pro y PRO, respectivamente. Por último, concluimos que, mientras que la identificación y la asignación del contenido de pro/PRO configuran dos operaciones distintas en chino, en español implican una sola.

\section{INTRODUCCIÓN}

En Chomsky (1981), pro y PRO se distinguen en su distribución y sus rasgos inherentes. La existencia de $P R O$ es universal y está limitada a la posición de sujeto de una cláusula infinitiva, como se señala en (1). Por otra parte, pro no aparece universalmente. En lenguas como el español, por ejemplo, debido a la rica concordancia flexiva del verbo conjugado, que permite recuperar o identificar la referencia de un sujeto elíptico, es posible que el pronombre se omita en una oración, tal como se demuestra en (2) y (3): 
(1) Juan quiere $P R O$ venir mañana.

(2) Juan cree que pro vendrá mañana.

(3) Juan quiere que pro venga mañana.

El chino constituye otra de las lenguas que también permiten la omisión del pronombre. Pero, en este caso, la hipótesis de la «Concordancia Rica» (Taraldsen 1978) no funciona ya que no es posible encontrar ningún tipo de concordancia morfológica en dicha lengua. Xu (1986) y Huang $(1984,1989)$ ofrecen diferentes propuestas para dar cuenta de los fenómenos observados que tienen relación con las categorías vacías en chino. El propósito del presente trabajo es revisar las teorías propuestas por Xu (1986) y Huang $(1984,1989)$. El contenido se organiza como sigue. Comentamos la CVL (Categoría Vacía Libre) de Xu (1986) en la sección 2 . En las secciones 3 y 4, señalamos cómo funciona la Regla de Control Generalizado propuesta por Huang $(1984,1989)$ en una lengua como el español en la que existen ambas categorías vacías, pro y PRO. Además, presentamos pruebas del español para apoyar la viabilidad de dicha regla. En la sección 5 , aducimos argumentos en contra del análisis semántico propuesto para resolver el problema del control libre causado por los verbos del tipo decir en español. En la sección 6, estudiamos los problemas relacionados con el control libre y la referencia arbitraria de las categorías vacías. Por último, se presentará la conclusión en la sección 7.

\section{CVL DE XU (1986) Y PROBLEMAS}

\subsection{CVL}

Tras considerar los estudios anteriores, Xu (1986) propone una nueva categoría, la Categoría Vacía Libre (CVL), que es una categoría que no tiene ningún rasgo específico que explique los elementos vacíos en chino. Su propuesta se basa en que los elementos vacíos en chino no pueden ser identificados por ninguno de los cuatro tipos de categoría vacía definidos por Chomsky (1982), es decir, «la traza de $S N$ », «la traza de $Q U$ » (variable), $P R O$ o pro, como se ilustra en (4), donde el elemento vacío no puede ser $P R O$ porque necesita estar controlado por un sujeto en la cláusula principal. El elemento vacío en (5) no puede ser pro porque no se comporta como un pronombre visible que pueda referirse a un $S N$ cuantificador:

(4) Xiaozhang ${\text { daying } t a / e_{i / j}}$ xingqisan yiqian gei huixin

Xiaozhang prometer él miércoles antes dar contestar-carta

'Xiaozhang (le) prometió que contestaría la carta antes del miércoles'.

(5) meiyouren, xiwang $e_{\mathrm{i}} /{ }^{*} t a_{\mathrm{i}}$ neng xingfu

nadie desear él poder feliz

'Nadie desea poder ser feliz./Nadie desea que él pueda ser feliz'. 


\subsection{Problemas}

Con respecto a la CVL de Xu (1986), en primer lugar, indicaremos que lo que propone $\mathrm{Xu}$ para la CVL no es adecuado. La manera para que uno llegue a su conclusión debe ser como sigue. Es verdad que el elemento vacío en los ejemplos estudiados no puede ser ninguno de los cuatro tipos de categoría vacía definidos por Chomsky. Por ejemplo, respecto de (4) con la referencia del subíndice «», a pesar de que el elemento vacio no necesita estar obligatoriamente controlado por el sujeto o el objeto principal como $P R O$ en inglés, Xu no trata de probar que puede ser otro tipo de categoría vacía tal como «la traza de QU», que es una variable. En cuanto a (5), los estudios anteriores han mostrado que los pronombres visibles e invisibles funcionan de forma distinta respecto a la referencia a un SN cuantificador. Por consiguiente, el elemento vacío que no se comporta exactamente igual que su correspondiente pronombre visible no debe, por lo tanto, ser inmediatamente excluido de ser un pronombre invisible. Debido a las dos razones que hemos mencionado, pensamos que lo que se propone en $\mathrm{Xu}$ (1986) debe rechazarse o bien requiere un estudio más profundo. Sin embargo, estamos de acuerdo con Xu (1986) en considerar que el contexto juega un papel importante para estudiar los elementos elípticos en chino debido a la característica de tópico que posee esta lengua. Dejando el aspecto extralinguístico para otra ocasión, nos concentraremos en el aspecto intralingüístico, analizando la Regla de Control Generalizado propuesta por Huang (1984, 1989) y su aplicación en español.

\section{LA RCG DE HUANG (1984)}

\subsection{Huang (1984): la Teoría de Control Generalizado original}

Huang, en su estudio del sujeto elíptico en chino, propone que el hecho de la omisión de pronombre puede explicarse bajo la Regla de Control Generalizado que determina la referencia de pro y $P R O$. Huang primero propone la Regla de Control Generalizado en 1984, y después, en 1989, la modifica. Vamos a comparar las dos versiones. Primero, la Regla de Control Generalizado (1984) se define como en (6):

(6) La Regla de Control Generalizado (1984) expresa:

Dé el mismo índice al pronombre vacío con un elemento mínimo más cercano. Según la RCG (1984), el elemento vacío en (7) puede ser un pronombre que tiene el mismo índice «» que el sujeto principal, el elemento sustantivo más cercano que lo c-comanda. En (8), el elemento vacío puede ser un pronombre o una variable: puede ser un pronombre con el índice «» por la misma razón que para (7), o puede ser una variable con el índice «» ligado por un tópico cero. Por lo tanto, la oración es ambigua.

(7) [ Zhangsan $_{\mathrm{i}}$ shefa Pro $_{\mathrm{i}}{ }^{\prime}$ bangmang wo]

Huang (38)

Zhangsan tratar ayudar yo

'Zhangsan trató de ayudarme'.

${ }^{\prime}$ Presentamos el pronombre de control generalizado con el símbolo Pro para distinguirse de pro y PRO. 
(8) Zhangsani shuo [Pro $/ \mathrm{vbl}_{\mathrm{j}}$ henxihuan Lisi ]

Huang (4a)

Zhangsan decir muy-querer Lisi

'Zhangsan dijo que querría a Lisi mucho.'

\subsection{Huang (1989): La Regla de Control Generalizado revisada}

La Regla de Control Generalizado (1984) se modifica y se presenta como la Regla de Control Generalizado Revisada (GCGR) que se expone en (9):

(9) Un pronombre vacío está controlado en su dominio de control (si existe uno)

La noción de dominio de control se define por Manzini (1983) y Nishigauchi (1984) en (10):

(10) Dominio de control (Manzini 1983 y Nishigauchi 1984)

$\alpha$ es el dominio de control para $\beta$ si y sólo si es la categoría mínima que satisface ambos (a) y (b).

(a) a es la O (Oración) o SN (Sintagma Nominal) más bajo que contiene

(i) $\beta$, o bien

(ii) la mínima categoría máxima que contiene $\beta$ (en adelante, $\operatorname{MCM}(\beta))$

(b) a contiene un SUJETO accesible a $\beta$.

Huang (1989: 194) indica que, según (10):

Un pro/PRO $(=\beta)$ tiene, a lo más, dos potenciales dominios de control: la $\mathrm{O}$ o el SN más bajo que contiene $\beta$ y la $\mathrm{O}$ o el SN más bajo que contiene MCM $(\beta)$. Si sólo una de estas dos categorías contiene un SUJETO accesible, la categoría es el dominio de control. Si ambas tienen un SUJETO accesible, sólo la más baja puede definirse como el dominio de control. Si ninguna contiene un SUJETO accesible, $\beta$ no tiene ningún dominio de control.

Según la RCGR, al contrario de lo que ocurre en español, la $O$ que domina inmediatamente el pro no es su dominio de control porque no existe un SUJETO accesible, que es la concordancia en esta $O$ mínima. A pesar de que el pro en chino no puede ser aceptado de la misma forma que en español al presentar la concordancia, es posible que se pueda aceptar como un pro por analogía con $P R O$. La situación es parecida a la ocurrencia de $P R O$ en la posición de sujeto de un verbo infinitivo cuya $\mathrm{O}$ mínima tampoco es un dominio de control potencial. Por consiguiente, ejemplos tales como (7) y (8) pueden tener la estructura de (10):

(10) $\left[\mathrm{O} \ldots \mathrm{S}_{\mathrm{i}} \ldots\left[\mathrm{O} \mathrm{O}^{\prime}\left[\mathrm{O} \ldots\right.\right.\right.$ pro/PRO $\left.\left.\left.\mathrm{PR}_{\mathrm{i}} \ldots\right]\right]\right]$ 
4. APLiCACión DE LA RCGR EN EL ESPAÑOL

\section{1. pro y PRO en español}

Según la RCG, en español, en una cláusula sustantiva como (11) y (12), un sujeto pro tiene su $\mathrm{O}$ en el nivel más bajo como su dominio de control porque es la $\mathrm{O}$ mínima que contiene pro y tiene un SUJETO accesible (la concordancia), como se exhibe en (13). En los ejemplos (11) y (12), la flexión de concordancia en un verbo conjugado es suficientemente rica para permitir recuperar los aspectos importantes del sujeto omitido, que puede ser un pronombre con los rasgos de la tercera persona, singular y masculino $(e ́ l)$, de la tercera persona, singular y femenino (ella), o de la segunda persona de tratamiento formal, singular ( usted). Como la concordancia es bastante rica para controlar pro, las oraciones (11) y (12) son gramaticales. La RCGR predice que el español permite la omisión del pronombre porque un sujeto pro siempre tiene un dominio de control en el que el pronombre invisible se puede controlar apropiadamente.

(11) Juan ${ }_{i}$ cree que pro ${ }_{i}$ está enfermo.

(12) Juan ${ }_{i}$ dijo que pro ${ }_{i}$ vio a Ana.

(13) $\left[\mathrm{O} \ldots \mathrm{S}_{i} \ldots\left[\mathrm{O}^{\prime}\left[\mathbf{O}\right.\right.\right.$ pro $\left.\left.\left._{i} \ldots\right]\right]\right]$

Por otro lado, según la RCGR, la oración (14) tiene la estructura (15) en la que $P R O$ tiene dos dominios de control potenciales, la $\mathrm{O}$ subordinada que contiene $P R O$ y la $\mathrm{O}$ principal que mínimamente contiene su $\mathrm{MCM}\left(=\mathrm{O}^{\prime}\right)$. Como sólo en la $\mathrm{O}$ de un nivel más alto, no en la $\mathrm{O}$ de un nivel más bajo, se encuentra la concordancia y, por lo tanto, contiene un SUJETO accesible, sólo la O más alta se define como el dominio de control para $P R O$ en (14):

(14) Juan ${ }_{\mathrm{i}}$ quiere $P R O_{\mathrm{i}}$ estar aquí.

(15) $\left[\mathrm{O} \ldots \mathrm{S}_{\mathrm{i}} \ldots\left[\mathrm{O}^{\prime}\left[\mathrm{O} P R O_{\mathrm{i}} \ldots\right]\right]\right]$

\subsection{Similitudes de pro y $P R O$}

A través de la revisión de los ejemplos españoles estudiados anteriormente, se puede hacer una observación: cuando los índices referenciales son idénticos en dos oraciones, ambas oraciones pueden tener el mismo significado (caso de 16 y 17). Ocurre lo mismo para los ejemplos (18) y (19). Es decir, el elemento elíptico puede ser pro en una oración finita tal como (16) o $P R O$ en una oración infinitiva tal como (17) con la misma interpretación. Esto mismo también se puede aplicar a las oraciones (18) y (19).

(16) Juan ${ }_{\mathrm{i}}$ cree que pro ${ }_{\mathrm{i}}$ vendrá mañana.

(17) Juan cree $P R O_{i}$ venir mañana.

(18) Juan ${ }_{i}$ dijo que pro ${ }_{i}$ está enfermo.

(19) Juan dijo $P R O_{\mathrm{i}}$ estar enfermo. 
El chino difiere del español en que no existe la concordancia flexiva ni el complementante; sin embargo, el hecho de que no haya flexión de concordancia no significa que la oración deba ser infinitiva. Como no está claro si la $\mathrm{O}$ mínima es finita o no, las estructuras (13) y (15) pueden aplicarse a los ejemplos chinos comportando el mismo significado que mencionamos anteriormente: la misma interpretación puede deducirse de dos estructuras con el mismo índice referencial del elemento vacío. Por lo tanto, las pruebas aportadas por los ejemplos españoles apoyan el argumento de tratar pro y $P R O$ como la misma categoría vacía bajo la RCGR. Las estructuras de los ejemplos chinos pueden ser paralelas a las del español: las oraciones (7) y (8) son paralelas a (16)-(19), que pertenecen al control de sujeto.

En las siguientes secciones, estudiaremos dos problemas relacionados con la RCGR.

5. ARGUMENTOS EN CONTRA DE LA SUGERENCIA RELACIONADA CON EL PROBLEMA DE CONTROL LIBRE PARA LOS VERBOS DEL TIPO DECIR

Después de aplicar la RCGR en español, un problema que debe estudiarse es la estructura de la oración (8) con la interpretación del índice $\alpha_{i} »$, que es exactamente igual a la de la oración de control obligatorio en (14). En la estructura de (14), como se señala en (15), el complemento «pro/PRO-sujeto» escoge la $O$ principal como su dominio de control. No obstante, aunque un pro/PRO tiene que estar controlado en (14), un pro/PRO en el ejemplo español (20) (revisión de (12)) y en el ejemplo chino (21) (revisión de (8)) puede aparecer sin control.

(20) Juan ${ }_{\mathrm{i}}$ dijo que $p r o / P R O_{\mathrm{i}, \mathrm{j}}$ vio a Ana.

(21) Zhangsan ${ }_{\mathrm{i}}$ shuo [pro/PRO $O_{\mathrm{i}, \mathrm{j}}$ kanjian Lisi le]

Zhangsan decir ver Lisi LE

'Zhangsan dijo que vio a Lisi'.

Con respecto a las propiedades del control, Huang (1989: 200) propone que la diferencia entre las dos oraciones chinas, (7) por un lado y (8) por otro, tiene que ver con la selección de los verbos principales. Los verbos try (tratar), persuade (convencer), permit (permitir) y order (mandar) requieren el control obligatorio de sujeto para el pro/PRO-complemento mientras que el control obligatorio no existe para los verbos tales como shuo (decir), wen (preguntar) y zhidao (saber). Huang sugiere el análisis de complementación oracional siguiendo a Rosenbaum (1967). Según dicho análisis, el complemento O' de (14) es directamente dominado por el SV, como se señala en (22), mientras que existe un $\mathrm{SN}$ que domina la $\mathrm{O}^{\prime}$ en el ejemplo (20) y ocupa la posición que interviene el SV y la O', como se señala en (23).

(22) $[\mathrm{O} \ldots[\mathrm{SV} \ldots[\mathrm{O},[\mathrm{O} \mathrm{pro} / \mathrm{PRO} \ldots]]]]]$

(23) $\left[\mathrm{O} \ldots\left[\right.\right.$... $\mathrm{SV} . . .\left[\mathrm{SN}\left[\mathrm{O}^{\prime}[\mathrm{O}\right.\right.$ pro/PRO...]]]] 
Huang (1989: 201) explica que:

Un verbo tal como force (forzar) o try (tratar) subcategoriza directamente un complemento $O^{\prime}$, mientras que un verbo tal como say (decir) o know (saber) subcategoriza un SN. Entonces, la $\mathrm{O}^{\prime}$ en (22) es un caso de complementación de SV mientras que la de (23) es el caso de complementación de SN. La diferencia entre (22) y (23) se distingue por la obligatoriedad u opcionalidad de control que el pro- $P R O$ en cuestión selecciona. En (22), el pro/PRO tiene la O principal como su dominio de control mientras que el pro/PRO no tiene ningún dominio de control en (23). Eso debido a que, si el pro/PRO tuviera un dominio de control, tendría que ser la $O$ subordinada que lo contiene mínimamente o el SN que está en un nivel más bajo que el $\mathrm{SV}$ que contiene la $\mathrm{O}^{+}$mínima, o sea, su MCM. No obstante, ni la O subordinada ni el SN contiene un SUJETO accesible para el pro/PRO. Por consiguiente, en la estructura tal como (23), un pro/PRO no necesita ser controlado.

Asimismo, Huang (1989: 202) añade lo que sigue a favor de adaptar el análisis de Rosenbaum (1967):

Sólo los verbos de complementos de verbos tales como shuo (decir), zhidao (saber), etc. pueden ser pasivos o tener la construcción de seudogrieta, y si el pro/PRO-sujeto de una cláusula de complemento no está obligatoriamente controlado, la cláusula puede ser pasiva y tener la construcción de seudogrieta, etc.

Sin embargo, proponemos que la dicha sugerencia no se aplica a los ejemplos españoles porque, según el análisis, las oraciones (17) y (19) van a tener la estructura de (22), que obedece al control obligatorio. No obstante, esto es contrario al argumento del tipo verbal de shuo (decir), que tiene la estructura de (23). Además, vamos a ofrecer ilustraciones para probar que el argumento a favor de adaptar el análisis de Rosenbaum (1967) no logra distinguir verbos como decir de otros verbos de control obligatorio, tal como tratar, porque el tipo verbal de decir en español puede ser pasivo o tener la construcción de seudogrieta como lo que se propone para el control libre, tal como se señala en (24), (25), (26) y (27), y también puede tener los resultados agramaticales de pasivos y seudogrieta como se propone para el control obligatorio, como se muestra en (28), (29), (30) y (31).

(24) Que vio a Ana fue dicho.

(25) Lo que dijo fue que vio a Ana.

(26) Que está enfermo es creído. 
(27) Lo que Juan cree es que está enfermo.

(28) *El ver a Ana fue dicho.

(29) *Lo que Juan dijo fue ver a Ana.

(30) *El estar enfermo es creído

(31) *Lo que Juan cree es el estar enfermo.

\section{CONTROL LIBRE E INTERPRETACIÓN ARBITRARIA}

Otro problema que requiere más explicación se relaciona con la interpretación arbitraria. Según la RCGR (1989), el elemento en (7) está controlado en su dominio de control que es la oración completa que contiene la MCM y el SUJETO accesible, que es el sujeto en un nivel más alto. Aunque la categoría vacía en el ejemplo español (32) y el ejemplo chino (33) tiene un dominio de control potencial, la $O$, que inmediatamente domina la categoría vacía, no existe un SUJETO accesible. Por eso, la categoría vacía no tiene ningún dominio de control y su interpretación es libre, es decir, puede tener una interpretación arbitraria o un referente específico que va a recuperarse del discurso.

(32) $\left[\mathrm{El}{ }_{s} p r o / P R O_{\text {Arb }}\right.$ fumar] es malo para la salud.

(33) $\left[{ }_{s}\right.$ pro/PRO ${ }_{\text {Arb }}$ xiyan] you hai

Huang (46) fumar tener desventaja

'El fumar es malo para la salud'.

La RCGR (1989) parece mejor que la RCG (1984) al tratar los sujetos vacíos como el mismo tipo de categoría vacía, pro. Además, la RCGR (1989) da cuenta de la interpretación arbitraria de (32) y (33), mientras que la RCG (1984) no puede explicar tal caso. Por otro lado, Jaeggli (1989) propone el Principio de Identificación por la Concordancia para dar cuenta de las lenguas de sujeto elíptico, como se señala en (34):

(34) La Concordancia puede identificar una categoría vacía como pro temático si y sólo si la categoría que contiene la Concordancia rige la categoría vacía por Caso.

Recordemos que la RCGR (1989) predice que el pro/PRO de (7) está controlado por el sujeto de la cláusula principal en su dominio de control, y que el Principio de Identificación por Concordancia no puede explicar los pronombres vacíos en chino. Para explicar los pronombres vacíos en chino, el Principio de Identificación tiene que ser revisado, como se señala en (35):

(35) La Concordancia o un SN puede identificar una categoría vacía como un pro temático.

En realidad, la idea del Principio de Identificación revisado no es nueva. Huang (1989:186) expone que «la hipótesis de identificación es esencialmente correcta, pero necesita ser interpretada en una forma más amplia de la que se supone la teoría de la Concordancia». Sin embargo, algunos problemas se han dejado bajo el Principio de Identificación revisado: excepto para el pronombre vacío en (8) y (12), el pronombre va- 
cío en los ejemplos españoles (35)-(36) y los ejemplos chinos (37)-(38) no queda identificado por el SN que ocupa la posición de sujeto en un nivel más alto:

(35) Zhangsan dijo que el pro/PRO ${ }_{\text {Arb }}$ fumar es malo para la salud.

(36) Zhangsan dijo que no es necesario pro/PRO ${ }_{\text {Arb }}$ ir al trabajo los fines de semana.

(37) Zhangsan shuo $_{\mathrm{i}}$ ro/PRO ${ }_{\text {Arb }}$ xiyan you hai

Zhangsan decir fumar tener desventaja

'Zhangsan dijo que el fumar es mal para salud'.

(38) Zhangsan $_{\mathrm{i}}$ shuo zhmou pro/PRO ${ }_{\text {Arb }}$ bu bi shanban ${ }^{2}$

Zhangsan decir fin-de-semana no necesario ir-a-trabajar

'Zhangsan dijo que no es necesario ir al trabajo los fines de semana'.

La RGC (1984) puede superar el problema fácilmente al establecer que el sujeto vacío no está coindexado con nada y, por lo tanto, debe tratarse como una variable. No obstante, si adoptamos la RCGR (1989) como una regla más adecuada para determinar los sujetos elípticos en las dos lenguas, el español y el chino, tendremos que resolver el problema en cuestión. Está claro que la RCGR de Huang (1989) puede correctamente predecir lo que se observa en (35)-(38), donde el sujeto vacío no necesariamente está controlado en el complemento subcategorizado por el tipo de verbo decir español o shuo (decir) chino. Entonces, la pregunta (39) tiene que ser contestada:

(39) Si el pronombre vacío no se identifica por la Concordancia ni por el SN, ¿cómo se identifica el pro/PRO con el índice « « en (20) y (21)?

La RGCR (1989) concluye que el pronombre vacío que no tiene ningún dominio de control tiene la interpretación libre. Y la interpretación libre significa que puede tener una referencia arbitraria o un referente definido que va a recuperarse del discurso. Por consiguiente, para dar cuenta de la identificación de un sujeto vacío en (20) y (21), el Principio de Identificación (35) tiene que revisarse de la forma como se señala en (40):

(40) La Concordancia, el SN o el discurso puede identificar una categoría como pro temático.

Sin embargo, el Principio de Identificación revisado (40) sólo resuelve la mitad del problema. El problema relacionado con la identificación del sujeto elíptico que contiene el significado arbitrario todavía se deja sin solución, como se señala en (36) y (38). Una alternativa para resolver el problema de identificación de pro/PRO ${ }_{\mathrm{Arb}}$ en (36) y (38) es modificar el Principio de Identificación revisado (40) proponiendo la Hipótesis de Identificador Arbitrario, que expone que el sujeto vacío es identificado por un elemento arbitrario. Entonces, se plantea la pregunta (41) relacionada con el Principio de Identificación revisado:

(41) ¿Son la identificación de pro/PRO y la asignación de contenido a pro/PRO dos operaciones? 
Para contestar la pregunta (41), compararemos la construcción estudiada anteriormente con una construcción similar que presenta una interpretación ambigua en español, como (42):

(42) pro llaman a la puerta.

Según el Principio de Identificación por la Concordancia propuesto por Jaeggli (1989), los rasgos de la concordancia [+tercera persona, +plural] identifican la categoría vacía como pro en (42), sin importar si pro se interpreta como pronombre definido o arbitrario, como se indica en Jaeggli (1986). Por consiguiente, se confirma otra vez que la identificación y la asignación de contenido son dos operaciones diferentes en español. Volvamos al caso del chino (43), a saber: la cláusula subordinada del ejemplo (38).

(43) zhoumou $p r o / P R O_{\mathrm{Art} / \mathrm{i}}$ bu bi shanban

fin-de-semana no necesario ir-a-trabajar

'No es necesario ir al trabajo los fines de semana'.

La oración (43) se interpreta como una oración ambigua, como (42), ya que presenta las dos interpretaciones, la arbitraria y la definida. En (43), a pesar de que el el pro/PRO con el índice «» puede ser identificado por el discurso según el Principio de Identificación revisado (40), el pro/PRO con el índice " $A r$ " se identifica sólo por la hipótesis de Identificador Arbitrario. Si adoptamos la Hipótesis en cuestión, el Principio de Identificación revisado es igual a la asignación de interpretación en chino. El sujeto vacío, pro/PRO, se identifica por $\mathrm{X}$, que asigna el contenido de un elemento vacío. Pues, al contrario de lo que concluimos anteriormente para el español, la identificación y la asignación de contenido de pro/PRO van a ser la misma operación en el chino.

Si no adoptamos la Hipótesis de Identificador Arbitrario, tenemos que proponer que el pro/PRO en chino se identifica por el SN o por el discurso; si no, se asigna el contenido por la Regla de Interpretación Arbitraria. Con respecto a la interpretación arbitraria, sugerimos que la Regla de Interpretación Arbitraria propuesta por Rizzi (1986: 521), como se señala en (44), puede aplicarse para asignar la interpretación arbitraria. Por lo tanto, la identificación y la asignación del contenido de pro/PRO son dos operaciones distintas en chino:

(44) Interpretación arbitraria

Asigna Arb al rol directo de $\theta$.

\section{CONCLUSIÓN}

De lo estudiado, hemos señalado que el español puede, por un lado, ofrecer pruebas que apoyen la Regla de Control Generalizado Revisado propuesta por Huang (1989); sin embargo, por otro lado, causa problemas para los análisis de Huang (1989) y Rosenbaum (1967), donde se intenta distinguir el verbo decir de tratar según sus características verbales. Por lo menos, los análisis necesitan ser modificados, o se requiere hacer la propuesta de que el tipo verbal de decir en español se comporta de forma diferente a co- 
mo lo hace en otros idiomas. Sin embargo, en la propuesta posterior se refuta su contribución con respecto al apoyo para la Regla de Control Generalizado. Además, modificamos el Principio de Identificación de Jaeggeli (1989) y aplicamos la Regla de Interpretación Arbitraria propuesta por Rizzi (1986) para resolver el problema sobre el control libre y la interpretación arbitraria del pro/ $P R O$, respectivamente. Por último, concluimos que la identificación y la asignación del contenido de pro/PRO son dos operaciones en chino mientras que es sólo una en español.

\section{REFERENCIAS BIBLIOGRÁFICAS}

Campos, H. (1992). «Silent Objects \& Subjects in Spanish», en H. Campos y Martínez-Gil (Eds.). Current Studies in Spanish Linguistics. Washington D.C.: Georgetown University Press.

CHOMSKY, N. (1981). Lectures on Government and Binding. Dordrecht: Foris.

- (1982). Some Concepts and Consequences of the Theory of Government and Binding. Cambridge: MIT Press.

- (1988). Language and Problem of Knowledge. Cambridge: MIT press.

Hernanz, M. L. y Brucat, J. M. (1987). La Sintaxis. La oración simple. Barcelona : Crítica.

HuAng, C.T. (1984). «On the Distribution and Reference of Empty Pronouns», Linguistic Inquiry 15: $531-574$.

- (1989). «Pro-drop in Chinese: a Generalized Control Theory», en O. Jaeggli y K. Safir. (Eds.). The Null subject Parameter. Dordrecht: Kluwer.

- (1991). «Remarks on the Status of the Null Object», en R. Freidin (Ed.). Principles and Parameters in Comparative Grammar. MIT Press.

JAEGGLI, O. (1986). «Arbitrary Pronominals», Natural Language and Linguistic Theory 4: 43-76. JAEGGLI, O. y Safir, K. (1989). «The Null Subject Parameter and Parametric Theory», en O. Jaeggli y K. Safir. (Ed.). The Null Subject Parameter. Dordrecht: Kluwer Academic Publishers. Manzinı. R. (1983). «On Control and Control Theory», Linguistic Inquiry 14: 421-446.

MeI, K. (1991). «On Generalized Control» (ms., National Tsing Hua University).

NiSHiGaUCHI, T. (1984). «Control and the Thematic Domain», Language 60: 215-250.

Rizzi, L. (1986). «Null Objects in Italian and the Theory of pro», Linguistic Inquiry 17: 501-558. Rosenbaum, P. S. (1967). The Grammar of English Predicate Complement Constructions. Cambridge, MA: MIT Press.

TARALDSEN T. (1978). «On the NIC, Vacous Application, and the That-trace Filter» distribuido por Indiana University Linguistics Club.

XU, L. (1986). «Free Empty Category», Linguistic Inquiry 17: 75-93. 